\title{
Long-term safety of icotinib in patients with non-small cell lung cancer: a retrospective, real-world study
}

\author{
Wen Zhang ${ }^{1}$, Yiping Zhang ${ }^{2}$, Qiong Zhao ${ }^{3}$, Xiguang Liu ${ }^{4}$, Likun Chen ${ }^{5}$, Hongming Pan ${ }^{6}$, Yuping Li $^{7}$, \\ You Lu ${ }^{8}$, Jianjin Huang ${ }^{9}$, Zhe Zhang ${ }^{10}$, Kaiqi Du ${ }^{11}$, Suning Zhang ${ }^{12}$, Tao $\mathrm{Li}^{13}$, Liqin Lu ${ }^{14}$, Guangmao $\mathrm{Yu}^{15}$, \\ Yang Wang ${ }^{16}$, Xiaobin Yuan ${ }^{16}$, Min Yang ${ }^{16}$, Yongbin Ma ${ }^{16}$, Fenlai Tan ${ }^{16}$
}

${ }^{1}$ Department of Cardiothoracic Surgery, the First Affiliated Hospital of People's Liberation Army General Hospital (304 Hospital), Beijing 100048, China; ${ }^{2}$ Department of Medical Oncology, Zhejiang Cancer Hospital, Hangzhou 310022, China; ${ }^{3}$ The Affiliated Shulan Hospital, Medical School of Zhejiang Shuren University, Hangzhou 310000, China; ${ }^{4}$ Department of Oncology, the Affiliated Hospital of Qingdao University, Qingdao 266000, China; ${ }^{5}$ Department of Medical Oncology, Sun-Yet San University Cancer Center, Guangzhou 510060, China; ${ }^{6}$ Department of Oncology, Sir Run Run Shaw Hospital, Zhejiang University, School of Medicine, Hangzhou 310016, China; ${ }^{7}$ Department of Respiratory Medicine, the First Affiliated Hospital of Wenzhou Medical University, Wenzhou 325000, China; ${ }^{8}$ Thoracic Cancer Department, West China Hospital, Sichuan University, Chengdu 610041, China; 'Department of Oncology, the Second Hospital Affiliated to Zhejiang University School of Medicine, Hangzhou 310009, China; ${ }^{10}$ Department of Thoracic Surgery, Qingdao Municipal Hospital, Qingdao 266011, China; ${ }^{11}$ Department of Thoracic Surgery, Chinese People's Armed Police Force, Zhejiang Corps Hospital, Hangzhou 314000, China; ${ }^{12}$ Department of Thoracic Surgery, Shengjing Hospital, China Medical University, Shenyang 110004, China; ${ }^{13}$ Radiation Therapy Department, Sichuan Cancer Hospital \& Institute, Chengdu 610041, China; ${ }^{14}$ Department of Oncology, Zhejiang Provincial People's Hospital, Hangzhou 310014, China; ${ }^{15}$ Department of Cardio-Thoracic Surgery, Shaoxing People's Hospital, Shaoxing 312000, China; ${ }^{16}$ Betta Pharmaceuticals Co., Ltd, Hangzhou 311100, China

Contributions: (I) Conception and design: W Zhang, F Tan; (II) Administrative support: Y Wang, X Yuan, M Yang, Y Ma, F Tan; (III) Provision of study materials or patients: W Zhang, Y Zhang, Q Zhao, X Liu, L Chen, H Pan, Y Li, Y Lu, J Huang, Z Zhang, K Du, S Zhang, T Li, L Lu, G Yu; (IV) Collection and assembly of data: W Zhang, Y Zhang, Q Zhao, X Liu, L Chen, H Pan, Y Li, Y Lu, J Huang, Z Zhang, K Du, S Zhang, T Li, L Lu, G Yu; (V) Data analysis and interpretation: W Zhang, Y Wang, X Yuan, M Yang, Y Ma, F Tan; (VI) Manuscript writing: All authors; (VII) Final approval of manuscript: All authors.

Correspondence to: Fenlai Tan, MD, PhD. Betta Pharmaceuticals Co., Ltd., 355 Xingzhong Road, Hangzhou 311100, China.

Email: tanfenlai@hotmail.com.

Background: Lung cancer is a global health problem with a high mortality, and the development of target therapy has led to a revolution in the treatment of lung cancer in recent years. Favorable efficacy and safety of icotinib have been demonstrated in patients with non-small cell lung cancer (NSCLC). Currently, minimal data are available to describe the long-term safety of icotinib in NSCLC patients.

Methods: We reviewed the safety data from 1,321 advanced NSCLC patients who were treated with icotinib. The primary endpoint was the long-term safety, defined as any adverse drug reactions (ADRs) occurred after 6 months of icotinib administration.

Results: Fewer ADRs were noticed over 6 month administration of icotinib than within 6 months in overall population $(24.3 \%$ vs. $65.4 \%)$, and elderly patients (23.6\% vs. $66.9 \%)$. The majority of ADRs were grade 1-2 in severity over 6 month exposure of icotinib in overall population as well as elderly patients. In overall population, the most common ADRs of icotinib during long-term use were rash (16.4\%) and diarrhea (5.3\%), while the incidences were $31.8 \%$ and $13.2 \%$ in the induction period, respectively. In elderly population, the most common ADRs of icotinib during long-term use were rash (15.7\%) and diarrhea (4.7\%), while the incidences were $27.8 \%$ and $14.9 \%$ in the induction period, respectively, and more inching was observed in the induction period as compared with long term use (6.3\% vs. $0.3 \%)$.

Conclusions: There was an evidence of decreased frequency of icotinib-induced ADRs over time, and icotinib was well-tolerated in elderly NSCLC patients.

Keywords: Epidermal growth factor receptor (EGFR); icotinib; long-term safety; non-small cell lung cancer (NSCLC); tyrosine kinase inhibitor (TKIs) 
Submitted Jun 13, 2019. Accepted for publication Dec 23, 2019.

doi: $10.21037 /$ jtd.2019.12.115

View this article at: http://dx.doi.org/10.21037/jtd.2019.12.115

\section{Introduction}

Lung cancer has become a particular public health problem in China, which is also the leading cause of cancerrelated death worldwide (1). Platinum-based combination chemotherapy is considered the standard of care for patients with advanced non-small cell lung cancer (NSCLC) $(2,3)$. The application of pemetrexed or bevacizumab as a component of the first-line and maintenance therapy conferred prolonged survival for patients with nonsquamous NSCLC (4-7). More recently, the identification of tumor-driven genes in lung cancer offered precision and personalized therapy for NSCLC patients. Mutations in the epidermal growth factor receptor (EGFR), one of the most important molecule biomarkers in NSCLC, strongly confer sensitivity to EGFR tyrosine kinase inhibitors (EGFR TKIs). First- and second-generation EGFR TKIs, gefitinib, erlotinib, and afatinib, have currently been introduced into clinical setting and produced durable responses in patients harboring EGFR activating mutations, with a progressionfree survival (PFS) of appropriately 8.4-13.1 months, and an overall survival (OS) of 21.6-30.9 months (8-13).

Icotinib is a first-generation, reversible, selective EGFR TKI, which is well-tolerated in a dose range of 100 to $500 \mathrm{mg}$ according to its dose-escalating studies $(14,15)$. The recommended dosage of icotinib (e.g., $125 \mathrm{mg}$ thrice per day) was established by a phase 3 study, in which significantly lower incidence of adverse reactions $(60.5 \%$ vs. $70.4 \%$ ) was documented in the icotinib arm than the control arm, with no treatment-related deaths or serious adverse reactions like interstitial lung disease (ILD) reported (16). A phase 4 , real-world study assessed the safety and tolerability of icotinib in 6087 NSCLC patients, and found that 31.5\% of patients experienced at least 1 adverse drug reactions (ADRs) of any grade, with rash (17.4\%) and diarrhea (8.5\%) the most common ones (17). However, current clinical trials are rarely focused on the long-term safety of icotinib, which is of significant importance because lung cancer is becoming a chronic disease and long-term use of icotinib can reasonably be expected. In addition, elderly patients with advanced NSCLC are less likely to be referred for surgery or chemotherapy than target therapy, and the safety profile of icotinib in this special population should also be addressed.
In the present study, we reviewed the safety profile of advanced NSCLC patients including the elderly who received long-term (e.g., $>6$ months) administration of icotinib.

\section{Methods}

\section{Study design and population}

This was a retrospective analysis of pooled data from a named patients use (NPU) program for icotinib in multicenters across China. Patients were eligible if they had histologically or cytologically confirmed locally advanced or metastatic NSCLC, who were older than 18 years, and received icotinib (125 mg tablet, Betta Pharmaceuticals Co., Ltd, Zhejiang, China) for 6 months or longer, and were available for safety data. Participating physicians were requested to report all adverse events (AEs) via request forms, which also included additional data such as anonymized datasets, including age, sex, stage of disease, genetic tests, laboratory tests, previous surgery or chemotherapy, and tumor response. At enrollment of the NPU program for icotinib, patients were required to provide information about short-term ADRs, and then were followed up for long-term ADRs once per month. The primary objective was to evaluate the long-term safety of icotinib. Elderly patients were defined as participants aged 70 years or older.

\section{Assessments}

Baseline characteristics included demographic data, vital signs, histological type, stage of disease, genetic tests, laboratory tests, previous surgery or chemotherapy, and tumor response. Response to icotinib could be classified into objective response rate, which is defined as the sum of complete remission (CR) and partial response (PR), and disease control rate which is defined as the sum of CR, PR and stable disease (SD).

The primary endpoint was long-term safety, which was defined as any ADRs and laboratory values occurred after 6 months of administration of icotinib. The long-term ADRs were categorized into three subtypes: newly-occurred ADR, defined as ADRs only occurred after 6-month 
administration of icotinib; persistent ADR, defined as ADRs occurred in the induction period (defined as the period from the initial administration of icotinib to 6 months) and persisted; and reoccurred ADR, defined as ADRs occurred and recovered in the induction period, and relapsed after 6 month administration of icotinib. Short-term safety included any ADRs and laboratory values occurred within 6 month administration of icotinib. ADRs were graded by the National Cancer Institute (NCI) Common Terminology Criteria for Adverse Events version (CTCAE) 3.0, and coded by the Medical Dictionary for Regulatory Activities (MedDRA) version 12.1. Icotinib-related serious adverse events and treatment withdrawal were also recorded. Tolerability in elderly patients was also included as a safety concern.

\section{Statistical analysis}

Descriptive methods including frequency and percentage were used for analysis. A logistic regression analysis model was employed to identify risk factors associated with the incidence of long-term safety of icotinib; the factors included age, gender, the Tumor, Node, Metastasis (TNM) stage, pathology, the Eastern Cooperative Oncology Group (ECOG) performance status, smoking status, treatment line, and EGFR mutation status. Statistical variables were assessed using the Cochran-MantelHaenszel $(\mathrm{CMH})$ test. Two-sided $\mathrm{P}<0.05$ was considered statistically significant. We did all statistical analyses using SAS software (version 9.4).

\section{Results}

\section{Patient characteristics}

Between February 2014 and November 2014, 1,321 eligible patients with NSCLC were included in the analysis set (intention-to-treat population) for long-term safety assessment (defined as treatment with icotinib for at least 6 months). A total of 162 patients were excluded from the analysis of short-term (treated with icotinib for less than 6 months) safety due to incomplete data $(n=162)$, leaving 1,159 evaluable patients (per-protocol population) for shortterm safety assessment.

Baseline characteristics including age, gender, disease stage, histological subtype, ECOG score, etc. were summarized in Table 1. The median age was 63.0 years (range: 28-91 years). The majority of patients had stage
IIIB/IV disease (89.7\%), adenocarcinoma (94.0\%), and good performance (ECOG 0-1; 94.7\%). Eighty-two patients $(6.2 \%)$ had a history of surgery or radiotherapy, and were fully recovered from previous therapies before enrolment. The distributions for patients receiving icotinib as first-line, second-line, and multiple-line treatments were $37.2 \%, 35.6 \%$, and $11.9 \%$, respectively. A total of 591 out of 1,321 patients went through the EGFR mutation testing, of which 429 patients had identified EGFR status, the rest of patients had no testing results may probably be due to the accessibility of EGFR mutation testing in study sites. Exon 21 L858R (51.2\%) and exon 19 deletions (47.3\%) were the major types of EGFR mutations. In addition, a total of 382 elderly patients (70 years or older) were included in the analysis set, whose baseline characteristics were similar with the overall population with the exception of disease stage and previous chemotherapy; the number of patients with stage I-IIIA disease (17.3\% vs. $8 \%$ ), and received firstline icotinib (52.1\% vs. $37.2 \%)$ were higher in the elderly population versus the overall population.

\section{Icotinib exposure and ADR pattern}

At data cut-off, the median duration of exposure was 259 days [interquartile range (IQR), 208-311 days]. Forty-nine percent of these patients received at least 10 months of icotinib treatment. The distribution types of long-term toxicity in the overall population and elderly subpopulation were analyzed (Figure 1). In the overall population, newlyoccurred ADRs were the most frequently recorded (12.9\%), followed by persistent ADRs (7.9\%), and reoccurred ADRs (3.5\%). Similar trends of distributions were noted in the elderly subpopulation (newly-occurred ADRs: $14.4 \%$; persistent ADRs: 6.0\%; reoccurred ADRs: 3.1\%). Three patients had a dose interruption ( 2 were due to non-medical reasons, 1 was due to flu), none of which was associated with ADRs. This finding was consistent with other studies evaluating the safety of icotinib and suggesting that few patients (less than $2 \%$ ) in the icotinib group had their treatment interrupted due to $\operatorname{ADRs}(16,17)$. Moreover, no ILD or treatment-related death was seen in this study.

\section{Safety profile of long-term icotinib in overall NSCLC patients}

The incidence and severity of long-term and short-term ADRs in the overall population were summarized in Table 2. Overall, ADRs were less frequently reported after 6 
Table 1 Baseline characteristics of the study population

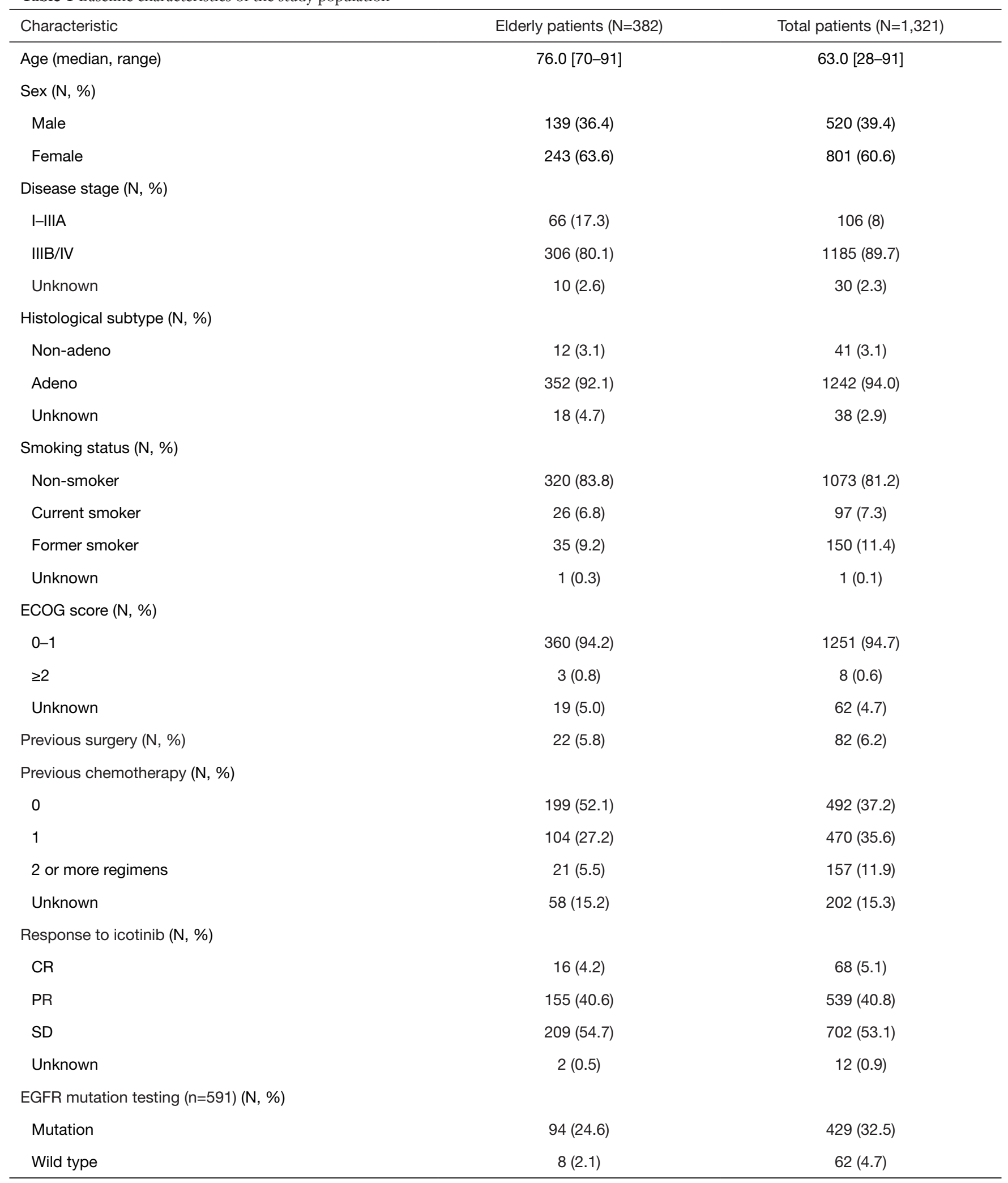

Adeno, adenocarcinoma; CR, complete remission; PR, partial response; SD, stable disease. 

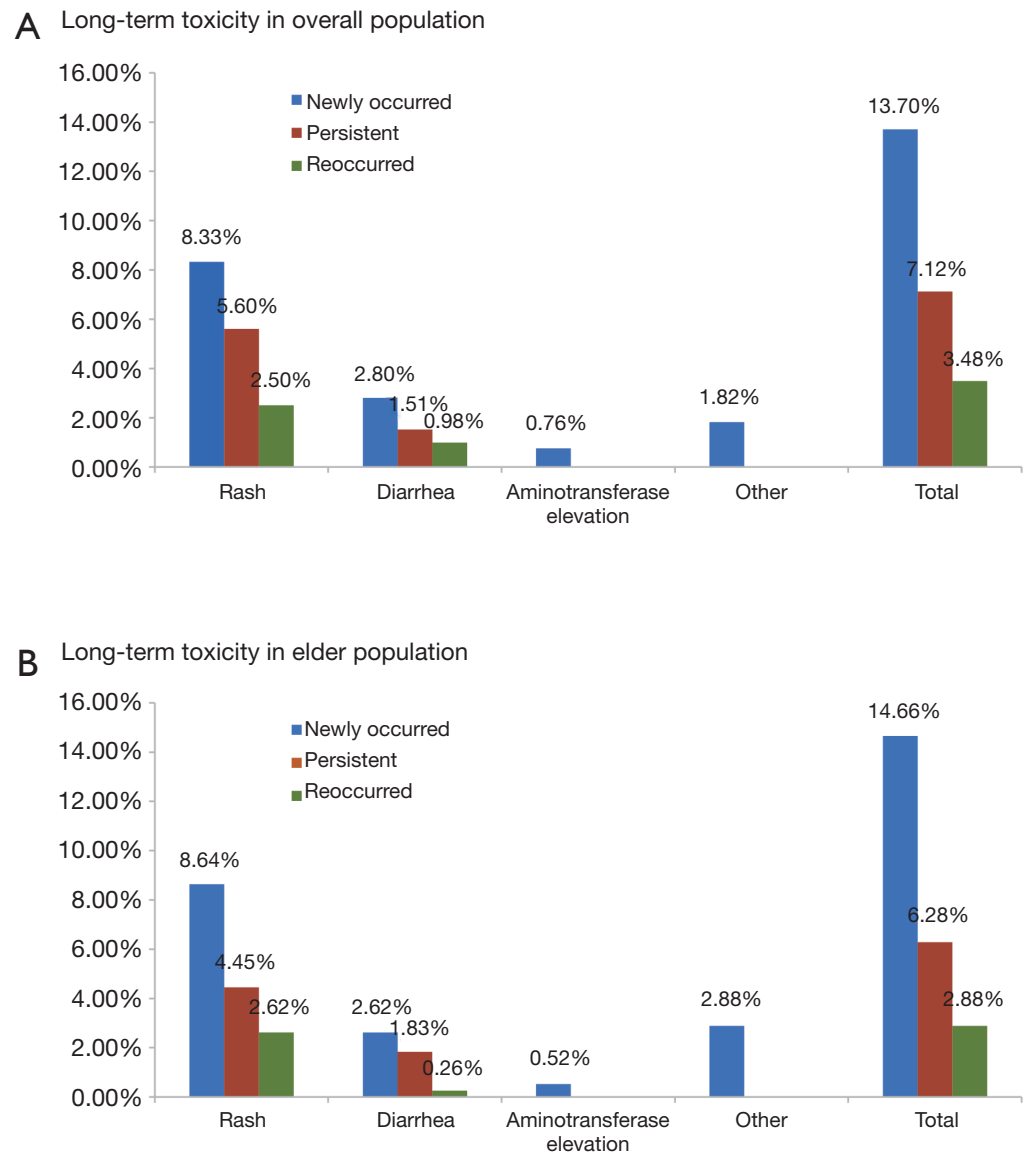

Figure 1 Long-term toxicity of icotinib in overall NSCLC population as well as elderly NSCLC patients in the study. The incidence and severity of ADRs in overall population (A) was similar to those in elderly patients (B). NSCLC, non-small cell lung cancer; ADR, adverse drug reaction.

months of icotinib treatment than that occurred in the induction period (e.g., within 6 months) $(24.3 \%$ vs. $65.4 \%$, $\mathrm{P}<0.0001)$. The most common ADRs were rash $(16.4 \%)$, and followed by diarrhea (5.3\%) during long-term exposure to icotinib, but their rates were $31.8 \%$ and $13.2 \%$ in the induction period, respectively. Aminotransferase elevation was not as common as rash or diarrhea, and was similar between induction period (1.6\%) and long-term exposure period $(0.8 \%)$. Most ADRs were of CTCAE grade 1 or 2 in severity either after or within 6 months of icotinib treatment. Few grade 3 or higher ADRs were observed in the induction period, and none was observed in the longterm period. The incidence and severity of long-term and short-term ADRs in patients with positive EGFR mutation $(\mathrm{N}=429)$ and wild type $(\mathrm{n}=62)$ were summarized in Tables 3,4, respectively. Results showed that fewer ADRs were observed in EGFR mutation positive ( $23.5 \%$ vs. $75.5 \%, \mathrm{P}<0.0001)$ and wild-type patients $(17.7 \%$ vs. $61.3 \%, \mathrm{P}<0.0001)$ during long-term exposure versus short-term exposure. No grade 3 or higher ADRs was seen during the induction period and the long-term period irrespective of regardless of EGFR mutation status.

\section{Safety profile of long-term icotinib in elderly NSCLC patients}

In the elderly NSCLC population, the incidence and severity of long-term and short-term ADRs were similar to those in the overall NSCLC population (Table 5, Figure 1). Fewer ADRs were noted after 6 months of icotinib treatment than that occurred in the induction period $(23.6 \%$ vs. $66.9 \%)$. During long-term exposure to icotinib, the most common ADRs were rash (15.7\%), and followed by diarrhea $(4.7 \%)$, while the incidences were $27.8 \%$ and $14.9 \%$ in the 
Table 2 The incidence and severity of short-term and long-term ADRs in overall population

\begin{tabular}{|c|c|c|c|c|c|c|c|}
\hline Variable & $\begin{array}{l}\text { Grade I } \\
(\mathrm{N}, \%)\end{array}$ & $\begin{array}{l}\text { Grade II } \\
(\mathrm{N}, \%)\end{array}$ & $\begin{array}{l}\text { Grade III } \\
(\mathrm{N}, \%)\end{array}$ & $\begin{array}{l}\text { Unknown } \\
(\mathrm{N}, \%)\end{array}$ & $\begin{array}{l}\text { Total } \\
(\mathrm{N}, \%)\end{array}$ & $\begin{array}{l}\text { Cochran-Mantel-Haenszel } \\
\text { none-zero correlation }\left(\chi^{2}\right)\end{array}$ & $P$ value \\
\hline Rash & & & & & & 51.9274 & $<0.0001$ \\
\hline Short-term & $318(27.4)$ & $39(3.4)$ & $1(0)$ & $11(0.9)$ & $369(31.8)$ & & \\
\hline Long-term & $175(13.2)$ & $34(2.6)$ & - & $8(0.6)$ & $217(16.4)$ & & \\
\hline Long-term & $61(4.6)$ & $5(0.4)$ & - & $4(0.3)$ & $70(5.3)$ & & \\
\hline Aminotransferase elevation & & & & & & 9.5334 & 0.0020 \\
\hline Short-term & $2(0.2)$ & $8(0.7)$ & - & $7(0.7)$ & $17(1.6)$ & & \\
\hline Long-term ${ }^{b}$ & $10(0.8)$ & $2(0.15)$ & - & $12(0.9)$ & $24(1.8)$ & & \\
\hline Total & & & & & & 225.3278 & $<0.0001$ \\
\hline Short-term & $587(50.6)$ & $58(5.0)$ & $2(0.17)$ & $111(9.6)$ & $758(65.4)$ & & \\
\hline Long-term & $255(19.3)$ & $42(3.2)$ & - & $24(1.8)$ & $321(24.3)$ & & \\
\hline
\end{tabular}

a , including pruritus $(n=50)$, skin exfoliation $(n=10)$, alopecia $(n=10)$, dry skin $(n=10)$, chapped skin $(n=5)$, paronychia $(n=3)$, skin discoloration $(n=3)$; ulcerative stomatitis $(n=21)$, appetite loss $(n=11)$, constipation $(n=9)$, nausea $(n=9)$, abdominal pain $(n=5)$, vomiting $(n=3)$, gastroesophageal reflux $(n=2)$, non-specific gastrointestinal disease $(n=2)$, dry mouth $(n=2)$, gum bleeding $(n=2)$, abdominal distension $(n=1)$; leucopenia $(n=2)$, hyperuricemia $(n=1)$, anemia $(n=1)$, thrombocytopenia $(n=1)$, increased alkaline phosphatase $(n=1)$, renal dysfunction $(n=3)$; laryngitis $(n=1)$, cough $(n=4)$, suffocation $(n=2)$, fever $(n=2)$, upper respiratory tract infection $(n=2)$, respiratory tract hemorrhage $(n=1)$, dyspnea $(n=1)$, increased sputum volume $(n=1)$; pain $(n=17)$, fatigue $(n=12)$, dizziness $(n=5)$, hyperhidrosis $(n=1)$, hemorrhage $(n=1)$, lower limb edema $(n=1)$, tachycardia $(n=1)$, and dizziness $(n=1) .{ }^{b}$, including pruritus $(n=4)$, paronychia $(n=1)$, skin exfoliation $(n=1)$, alopecia $(n=1)$; cough $(n=4)$, upper respiratory tract infection $(n=1)$, increased sputum $(n=1)$; dry tongue $(n=1)$, and pleural effusion $(n=1)$.

induction period, respectively. Of note, more inching was noticed in elderly NSCLC patients in the induction period than the long-term period (6.3\% vs. $0.3 \%)$.

\section{Predictive factors for long-term safety of icotinib}

Multivariate analyses were performed using the logistic regression model to estimate the predictive factors for longterm toxicity of icotinib in NSCLC patients (Table 6). The logistic regression analysis showed that male [odds ratio (OR) 1.347, 95\% CI: 1.03-1.76; $\mathrm{P}=0.0282]$ was predictive of occurrence of long-term toxicity. Despite failing to show a statistical significance, patients with poor ECOG PS (e.g., 2 or more) had a higher trend to develop long-term toxicity (OR 3.739, 95\% CI: 0.93-15.05; $\mathrm{P}=0.0634$ ). Rash was more likely to be seen in male than female after 6 months of icotinib treatment (OR 1.788, 95\% CI: 1.34-2.38; $\mathrm{P}<0.0001)$. The risk of getting transaminase elevation was associated with age (per increase of 1 year; OR $0.971,95 \%$ CI: 0.95-1.00; $\mathrm{P}=0.0303$ ), and ECOG PS $\geq 2$ (OR 9.813, 95\% CI: $1.92-50.10 ; \mathrm{P}=0.006)$. No clinical or pathologic factor was detected for diarrhea. Univariate analyses were performed to estimate the predictive factors for newlyoccurred adverse events, and no clinical or pathologic factor was detected (Table 7).

\section{Discussion}

The current study showed that long-term use of icotinib induced bearable ADRs as compared with short-term use. Most ADRs were newly-occurred type, and of CTCAE grade 1 in severity. During long-term exposure to icotinib, 
Table 3 The incidence and severity of short-term and long-term ADRs in EGFR mutation population (N=429)

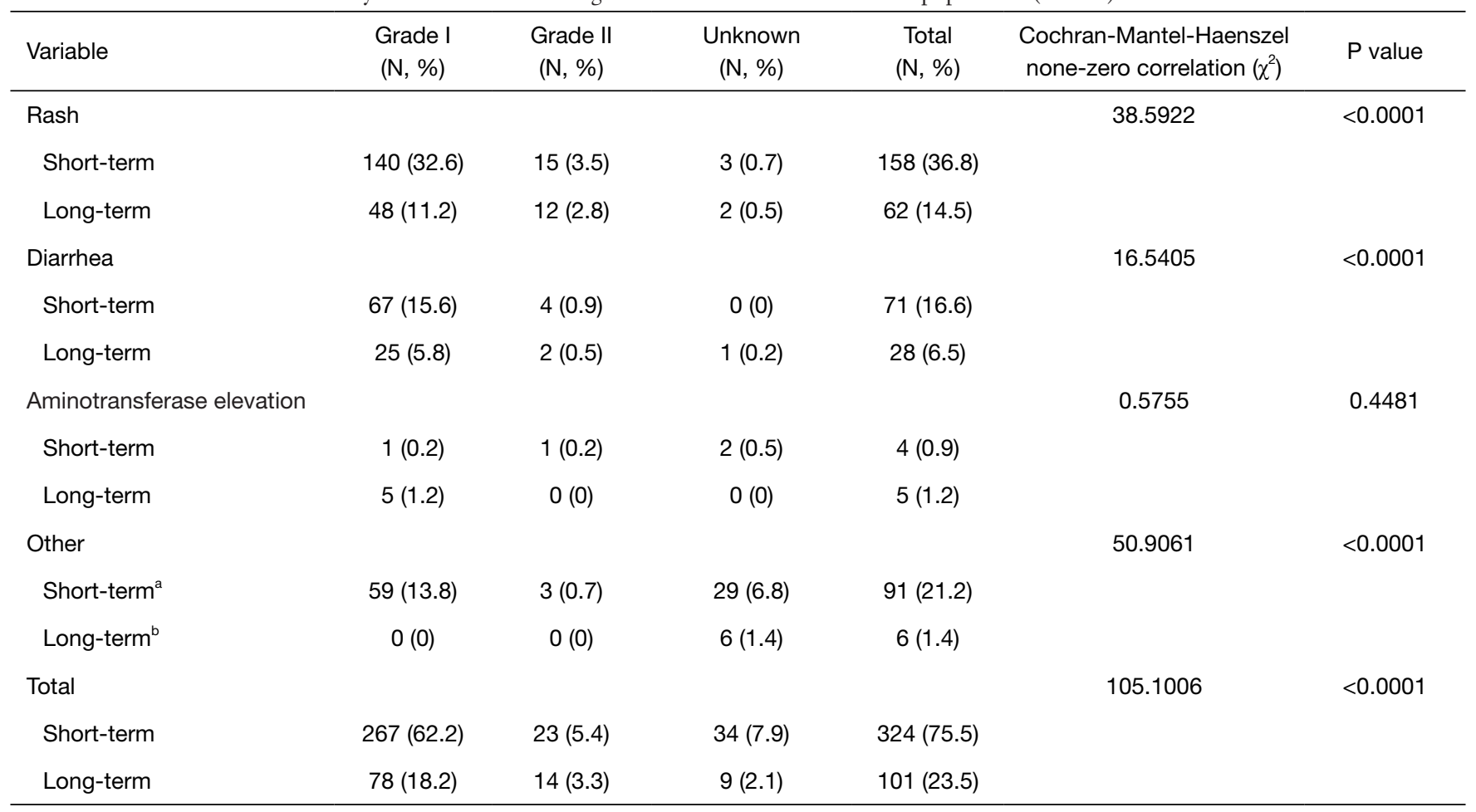

a: including pruritus $(n=18)$, ulcerative stomatitis $(n=10)$, appetite loss $(n=6)$, pain $(n=6)$, constipation $(n=9)$, dry skin $(n=4)$, unnormal labraotory testing $(n=4)$, chapped skin $(n=4)$, nausea $(n=3)$, vomiting $(n=3)$, vomiting $(n=3)$, alopecia $(n=3)$, fatigue $(n=3)$, abdominal pain $(n=2)$, cough $(n=2)$, headache $(n=2)$, leucopenia $(n=1)$, non-specific gastrointestinal disease $(n=1)$, abdominal distension $(n=1)$, dry mouth $(n=1)$, gastroesophageal reflux $(n=1)$, hemorrhage $(n=1)$, hyperuricemia $(n=1)$, paronychia $(n=1)$, increased alkaline phosphatase $(n=1)$, renal dysfunction $(n=1)$; skin exfoliation $(n=1)$, dizziness $(n=1)$, skin discoloration $(n=1)$, dyspnea $(n=1)$, and hyperhidrosis $(n=1)$. ${ }^{b}$ : including cough $(n=2)$, pruritus $(n=1)$, numbmess $(n=1)$, alopecia $(n=1)$, and pleural effusion $(n=1)$.

Table 4 The incidence and severity of short-term and long-term ADRs in wild-type EGFR population (N=62)

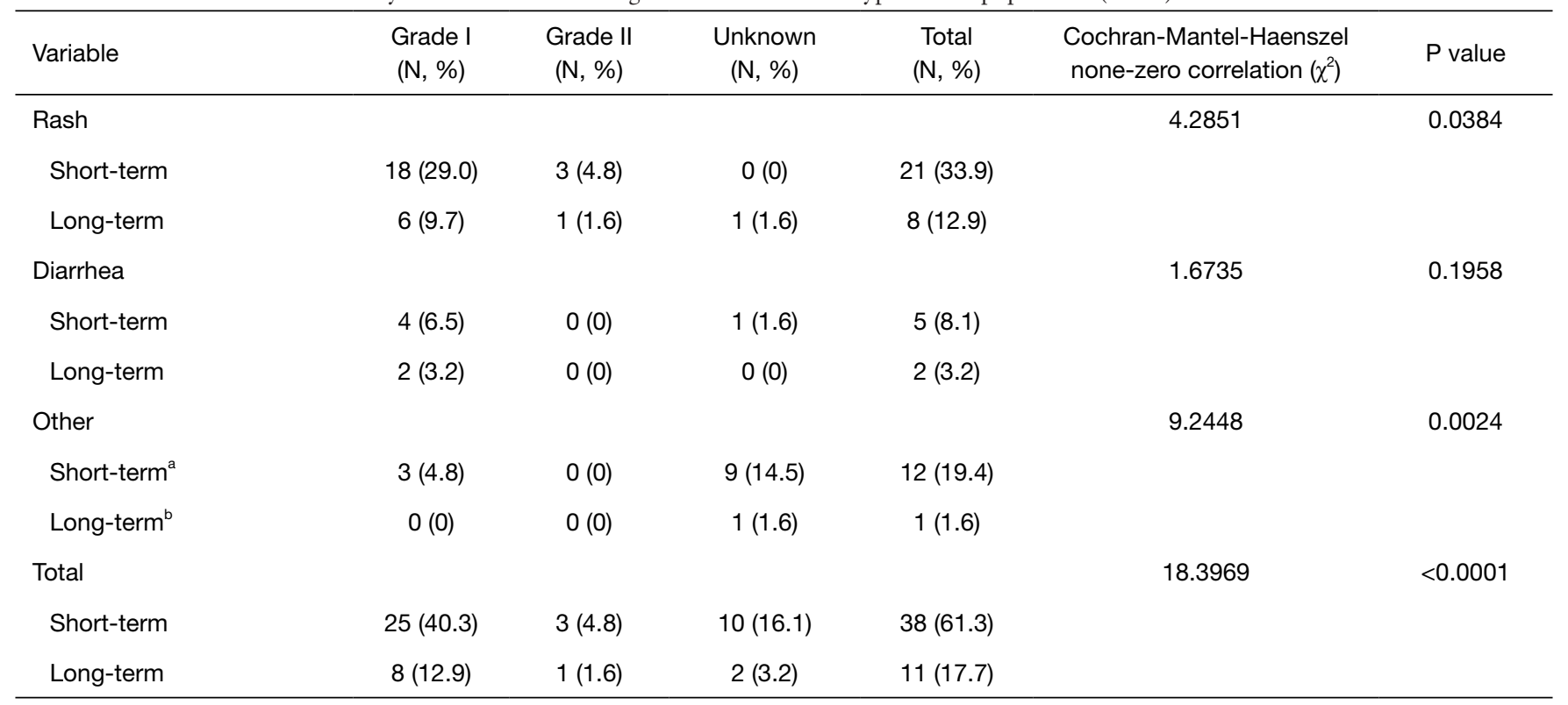

a , including pruritus $(n=4)$, dry skin $(n=2)$; constipation $(n=1)$, renal dysfunction $(n=1)$; fever $(n=1)$, pain $(n=1)$, fatigue $(n=1)$, headache $(n=1)$, including cough $(n=1)$. 
Table 5 The incidence and severity of short-term and long-term ADRs in elder patients

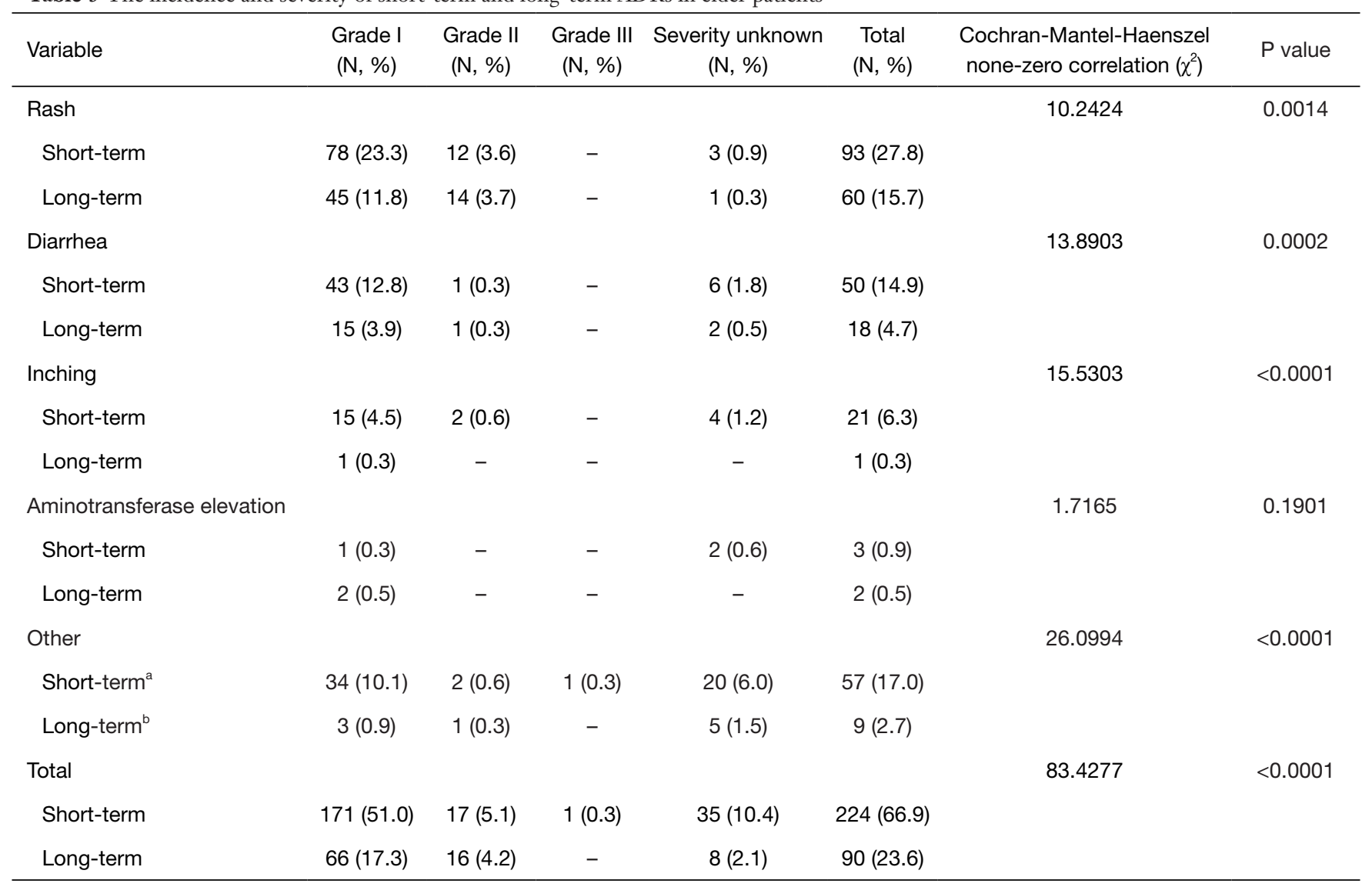

a , including alopecia $(n=5)$, skin exfoliation $(n=4)$, dry skin $(n=3)$, skin discoloration $(n=2)$, paronychia $(n=2)$, ulceration $(n=1)$; ulcerative stomatitis $(n=7)$, nonspecific appetite $(n=6)$, nausea $(n=3)$, vomiting $(n=1)$, dry mouth $(n=1)$, abdominal pain $(n=1)$; increased sputum volume $(n=1)$, upper respiratory tract infection $(n=1)$, cough $(n=1)$, suffocation $(n=1)$; renal dysfunction $(n=1)$, anemia $(n=1)$; headache $(n=1)$, partial numbness $(n=1)$, hyperhidrosis $(n=1)$; pain $(n=1)$, suffocation $(n=1)$, hyperhidrosis $(n=1)$, and fatigue $(n=5)$. ${ }^{b}$, including paronychia $(n=1)$, dry eye $(n=1)$, cough $(n=1)$, vomiting $(n=2)$., upper respiratory tract infection $(n=1)$, increased sputum volume $(n=1)$, and fatigue $(n=2)$. ADRs, adverse reactions.

the most common ADRs were rash and diarrhea. We also explored the potential predictors of long-term toxicity of icotinib in NSCLC patients, and found that male gender was a predictive factor of the occurrence of long-term toxicity. The safety profile of long-term use of icotinib in the elderly patients did not differ significantly from that in the overall population. The present study has added evidence about the safety of EGFR TKI during longterm use, which few studies have focused on. To the best of our knowledge, this is the largest study that evaluates the long term safety including distribution type, incidence, severity, predictive factors of icotinib, which could be more representative of patients seen in real-world daily oncology experience with icotinib.

Even though a lower incidence of systemic ADRs compared with standard chemotherapy was found, EGFR TKIs specific ADRs have been identified, particularly skin, gastrointestinal and hepatic toxicities $(8-13,18,19)$. However, long-term toxicities of EGFR TKIs have been rarely studied. With the discovery of the relationship between EGFR mutations and responsive population, more and more patients received long-term EGFR-TKI therapy (2.2-13.6 months) $(8-13,18,19)$; moreover, EGFR TKIs is now being tested as an adjuvant therapy for patients with early-stage disease (20). Therefore, the impact of chronic ADRs of icotinib is even more critical.

The frequency of ADRs arising from long-term use of icotinib was quite different from that of published studies of icotinib and other EGFR TKIs, since the latter mostly concerned early reactions. Early rash is reported to occur 
Table 6 Predictive factors for long-term toxicity of icotinib using multivariate analyses

\begin{tabular}{|c|c|c|c|c|c|c|c|c|c|}
\hline Variable & \multicolumn{3}{|c|}{ Overall incidence of ADR } & \multicolumn{3}{|c|}{ Rash } & \multicolumn{3}{|c|}{ Diarrhea } \\
\hline Elderly to non-elderly & 0.788 & $0.58-1.06$ & 0.1188 & 0.805 & $0.59-1.11$ & 0.1919 & 0.638 & $0.36-1.12$ & 0.1192 \\
\hline Male to female & 1.347 & $1.03-1.76$ & $0.0282^{*}$ & 1.788 & $1.34-2.38$ & $<0.0001^{*}$ & 0.787 & $0.48-1.28$ & 0.3364 \\
\hline Stage IIIB/IV to I-IIIA & 1.610 & $0.83-3.13$ & 0.1608 & 1.928 & $0.94-3.97$ & 0.0749 & 0.720 & $0.25-2.11$ & 0.5492 \\
\hline Current smoker to non-smoker & 0.957 & $0.57-1.60$ & 0.8676 & 1.045 & $0.60-1.81$ & 0.8750 & 0.840 & $0.33-2.14$ & 0.7151 \\
\hline Former smoker to non-smoker & 1.040 & $0.69-1.57$ & 0.8540 & 1.281 & $0.84-1.96$ & 0.2549 & 0.644 & $0.27-1.51$ & 0.3131 \\
\hline EGFR mutation: positive to negative & 1.560 & $0.76-3.18$ & 0.2216 & 1.168 & $0.55-2.48$ & 0.6855 & 2.594 & $0.61-11.08$ & 0.1980 \\
\hline ECOG score 2 to $0-1$ & 3.739 & $0.93-15.05$ & 0.0634 & 1.624 & $0.33-8.09$ & 0.5543 & 2.270 & $0.28-18.68$ & 0.4460 \\
\hline
\end{tabular}

*, indicates a statistically significant difference with a $\mathrm{P}$ value less than 0.05 . ADR, adverse reaction; OR, odd ratio; Cl, confidence interval.

Table 7 Predictive factors for newly-occurred long-term toxicity of icotinib using univariate analyses

\begin{tabular}{|c|c|c|c|}
\hline Male to female & 1.044 & $0.74-1.48$ & 0.8098 \\
\hline Stage IIIB/IV to I-IIIA & 1.547 & $0.62-3.86$ & 0.3492 \\
\hline Current smoker to non-smoker & 0.783 & $0.38-1.59$ & 0.4997 \\
\hline Former smoker to non-smoker & 0.914 & $0.53-1.59$ & 0.7486 \\
\hline EGFR mutation: positive to negative & 1.693 & $0.50-5.69$ & 0.3945 \\
\hline ECOG score 2 to $0-1$ & 2.667 & $0.53-13.34$ & 0.2323 \\
\hline
\end{tabular}

ADR, adverse reaction; OR, odd ratio; $\mathrm{Cl}$, confidence interval.

in $46.6-66.2 \%$ of patients on gefitinib $(8,9,19,21), 73-80 \%$ on erlotinib $(10,11,18)$, and $40 \%$ on icotinib $(16)$, whereas in our study it was presented in $16.4 \%$ of the patients after at least 6 month use of icotinib. The reduced rate of rash caused by icotinib in our study may be due to non-selected population (both EGFR-mutated and wild-type EGFR mutation) was included in our safety analysis. Diarrhea, another common ADRs caused by icotinib, has an incidence of $5.3 \%$ during long-term use, which was less than shortterm use of icotinib or other EGFR TKIs having an incidence of $19-57 \%$ (8-11,16,18-21). Possible mechanisms for EGFR TKIs-associated cutaneous toxicity included keratinocyte growth arrest and apoptosis, decreased cell migration, increased cell attachment and premature differentiation, and stimulation of inflammation $(22,23)$. However, the pathogenic mechanisms remain incompletely understood.

Different from other ADRs, similar incidence of transaminase elevation was seen between long-term and short-term use of icotinib (1.6\% vs. $0.8 \%)$. According to previous reports, administration of icotinib would induce transaminase elevation in $11.1 \%$ of patients (16), whereas $5-70.1 \%$ for gefitinib and $4-37 \%$ for erlotinib, respectively (8-11,24,25). Wang et al. reported drug-induced hepatic 
toxicity by long-term gefitinib administration in the treatment of NSCLC: 40 out of 101 patients (39.6\%) developed abnormal hepatic function, with a median onset time of 4 months (1-23 months) (26). In addition, $20 \%$ patients $(8 / 40)$ had persistent grade I hepatotoxicity until the last follow-up. This study also found that longer exposure to gefitinib (more than 14 months) was associated with higher incidence of hepatotoxicity $(52.0 \mathrm{vs} .27 .5 \%$; $\mathrm{P}=0.012$ ) (26). Dysfunctional CYP2D6 was reported to be associated with an increased incidence of gefitinibinduced hepatotoxicity (27-29). For icotinib, CYP3A1/2, CYP2C9/10 and CYP2E1 were the main enzymes involved in its metabolization (30), whether and how these enzymes work in the mechanism on icotinib-induced transaminase elevation is still unknown.

The long-term safety data of this study was compared to that of other first generation EGFR-TKI. The most common ADRs during long-term exposure to icotinib were rash and diarrhea according to this study. Based on previous reports, long-term use of gefitinib was generally well-tolerated in advanced NSCLC patients, rash was less frequently seen in gefitinib than icotinib ( $6 \%$ vs. 16.4\%), whereas, it was associated with a higher frequency of hepatotoxicity $(12.5 \%$, grade $1-2 ; 4 \%$, grade 3 or higher) as compared with icotinib $(0.8 \%$, all grade $1-2)(31,32)$. Most of the long-term toxicities related to erlotinib were considered bearable, paronychia, fatigue, and hair changes were reported, besides the well-known toxicities such as folliculitis and diarrhea (33). Erlotinib therefore had a different ADR pattern compared with icotinib, with a higher incidence of folliculitis, fatigue, keratoconjunctivitis, paronychia, and hair changes, which were barely seen in long term icotinib treatment. Among first generation EGFR-TKIs, diarrhea was a mutual long-term toxicity, mostly grade 1-2 in severity. Nevertheless, it is hard to make an explicit and direct comparison, due to different sample size and definition of long-term treatment among these studies.

It is noted that newly-occurred ADRs developed in as many as $12.9 \%$ in the study, some hypotheses may include that only patients treated for more than 6 months were enrolled, and we didn't take ADRs occurred before 6 month administration of icotinib and never appeared again (due to spontaneous regression or treatment) into account, in addition, patients treated for over 6 months have higher exposure, despite low accumulation, patients may develop cumulative toxicity due to patient- or tumor-related factors. It should be also noted that the number of patients decreases with increasing treatment cycles, which may affect the toxic rate. In general, icotinib and other EGFR TKIs have comparable safety. Since this is the first study that categorizes icotinib associated long-term safety into three types, it is hard to draw a conclusion that this phenomenon is unique in icotinib.

Potential predictors of long-term toxicity in NSCLC patients were explored, and we found that male gender was a predictive factor of occurrence of overall toxicity $(\mathrm{P}=0.0282)$. It might be attributed to that male patients are more likely to be lifetime smokers than female patients, and smoking habits may affect the occurrence of side effects induced by icotinib. It's worth mentioning that smoking status was not statistically significant in predicting the occurrence of overall toxicity (current smoker versus nonsmoker, $\mathrm{P}=0.8676$; former smoker versus non-smoker, $\mathrm{P}=0.8540)$ in this study. This may due to that more detailed information of smoking such as duration and intensity of smoking, and type of cigarettes consumed was not collected and analyzed. A previous study revealed that there was significant disparity between female and male in their adherence to medications, female were less likely to be adherent in use of medications and to receive the treatment recommended by clinical guidelines during long-term period (34). All together may imply the gender differences in predicting overall occurrence of long-term toxicity.

The present study has several limitations. First, we did not evaluate EGFR mutation status in all of the patients. A favorable clinical response to icotinib was found in lung cancer patient with positive EGFR mutation status, analysis of EGFR mutation status may better explain the appropriate patients for icotinib safety and treatment response. Second, this is a retrospective study, and lacks of assessment of quality of life and measurement of some key statistics. In addition, we didn't specifically analyze the long-term safety data between elderly patients and other-aged patients (e.g., younger patients). At last, we can't identify when each longterm ADR appeared during icotinib therapy since we failed to collect these data. However, the results of this study expand the current understanding of long-term toxicity caused by EGFR TKIs, which has been underestimated in previous studies, and deserves more attention since increasing patients have longer duration of EGFR TKI treatment.

In conclusion, long-term icotinib is well tolerated in both overall and elderly population. The ADR pattern and profile of icotinib during long-term use were similar to that during short-term exposure, but the incidence and severity were 
milder, indicating icotinib has low accumulated toxic effect and could be continually used until disease progression without major long-term safety or tolerability problems in clinical practice. Male was predictive of occurrence of longterm toxicity. Further studies are warranted to prospectively evaluate the safety during chronic use of icotinib.

\section{Acknowledgments}

We thank the patients, their families, and all of the investigators who participated in the study.

Funding: This work was supported by the Betta Pharmaceuticals Co., Ltd (Hangzhou, China), and partly supported by the grant from National Key Special Program for Innovative Drugs (grant number 2012ZX09101103).

\section{Footnote}

Conflicts of Interest: Fenlai Tan, Yang Wang, Xiaobin Yuan, Min Yang, and Yongbin Ma are salaried employees of Betta Pharmaceuticals Co., Ltd. The other authors have no conflicts of interest to declare.

Ethical Statement: The authors are accountable for all aspects of the work in ensuring that questions related to the accuracy or integrity of any part of the work are appropriately investigated and resolved. This study is a post-market survilance progromme for which IRB is not mandatory by local regulations, so we can't provide relevant details.

Open Access Statement: This is an Open Access article distributed in accordance with the Creative Commons Attribution-NonCommercial-NoDerivs 4.0 International License (CC BY-NC-ND 4.0), which permits the noncommercial replication and distribution of the article with the strict proviso that no changes or edits are made and the original work is properly cited (including links to both the formal publication through the relevant DOI and the license). See: https://creativecommons.org/licenses/by-nc-nd/4.0/.

\section{References}

1. Siegel RL, Miller KD, Jemal A. Cancer statistics, 2017. CA Cancer J Clin 2017;67:7-30.

2. Spiro SG, Rudd RM, Souhami RL, et al. Chemotherapy versus supportive care in advanced non-small cell lung cancer: improved survival without detriment to quality of life. Thorax 2004;59:828-36.

3. NSCLC Meta-Analyses Collaborative Group.

Chemotherapy in addition to supportive care improves survival in advanced non-small-cell lung cancer: a systematic review and meta-analysis of individual patient data from 16 randomized controlled trials. J Clin Oncol 2008;26:4617-25.

4. Scagliotti GV, Parikh P, Von P J, et al. Phase III study comparing cisplatin plus gemcitabine with cisplatin plus pemetrexed in chemotherapy-naive patients with advanced-stage non-small-cell lung cancer. J Clin Oncol 2008;26:3543-51.

5. Ciuleanu T, Brodowicz T, Zielinski C, et al. Maintenance pemetrexed plus best supportive care versus placebo plus best supportive care for non-small-cell lung cancer: a randomised, double-blind, phase 3 study. Lancet 2009;374:1432-40.

6. Sandler A, Gray R, Perry MC, et al. Paclitaxel carboplatin alone or with bevacizumab for non-small cell lung cancer. N Engl J Med 2006;355:2542-50.

7. Karayama M, Inui N, Fujisawa T, et al. Maintenance therapy with pemetrexed and bevacizumab versus pemetrexed monotherapy after induction therapy with carboplatin, pemetrexed, and bevacizumab in patients with advanced non-squamous non small cell lung cancer. Eur J Cancer 2016;58:30-7.

8. Maemondo M, Inoue A, Kobayashi K, et al. Gefitinib or chemotherapy for non-small-cell lung cancer with mutated EGFR. N Engl J Med 2010;362:2380-8.

9. Mitsudomi T, Morita S, Yatabe Y, et al. Gefitinib versus cisplatin plus docetaxel in patients with non-small-cell lung cancer harbouring mutations of the epidermal growth factor receptor (WJTOG3405): an open label, randomised phase 3 trial. Lancet Oncol 2010;11:121-8.

10. Zhou C, Wu YL, Chen G, et al. Erlotinib versus chemotherapy as first-line treatment for patients with advanced EGFR, mutation-positive non-small-cell lung cancer (OPTIMAL, CTONG-0802): a multicentre, open-label, randomised, phase 3 study. Lancet Oncol 2011;12:735-42.

11. Rosell R, Carcereny E, Gervais R, et al. Erlotinib versus standard chemotherapy as first-line treatment for European patients with advanced EGFR mutation-positive non-small-cell lung cancer (EURTAC): a multicentre, open-label, randomised phase 3 trial. Lancet Oncol 2012;13:239-46.

12. Yang JC, Shih JY, Su WC, et al. Afatinib for patients with lung adenocarcinoma and epidermal growth factor 
receptor mutations (LUX-Lung 2): a phase 2 trial. Lancet Oncol 2012;13:539-48.

13. Sequist LV, Yang JC, Yamamoto N, et al. Phase III study of afatinib or cisplatin plus pemetrexed in patients with metastatic lung adenocarcinoma with EGFR mutations. J Clin Oncol 2013;31:3327-34.

14. Hu S, Xie G, Zhang DX, et al. Synthesis and biological evaluation of crown ether fused quinazoline analogues as potent EGFR inhibitors. Bioorg Med Chem Lett 2012;22:6301-5.

15. Liu J, Wu L, Wu G, et al. A Phase I Study of the Safety and Pharmacokinetics of Higher-Dose Icotinib in Patients With Advanced Non-Small Cell Lung Cancer. Oncologist 2016;21:1294-5d.

16. Shi Y, Zhang L, Liu X, et al. Icotinib versus gefitinib in previously treated advanced non-small-cell lung cancer (ICOGEN): a randomised, double-blind phase 3 noninferiority trial. Lancet Oncol 2013;14:953-61.

17. Hu X, Han B, Gu A, et al. A single-arm, multicenter, safety-monitoring, phase IV study of icotinib in treating advanced non-small cell lung cancer (NSCLC). Lung Cancer 2014;86:207-12.

18. Shepherd FA, Rodrigues Pereira J, Ciuleanu T, et al. Erlotinib in previously treated non-small-cell lung cancer. N Engl J Med 2005;353:123-32.

19. Kim ES, Hirsh V, Mok T, et al. Gefitinib versus docetaxel in previously treated non-small-cell lung cancer (INTEREST): a randomized phase III trial. Lancet 2008;372:1809-18.

20. Kelly K, Altorki NK, Eberhardt WE, et al. Adjuvant erlotinib versus placebo in patients with stage IB-IIIA nonsmall-cell lung cancer (RADIANT): a randomized, doubleblind, phase III trial. J Clin Oncol 2015;33:4007-14.

21. Fukuoka M, Yano S, Giaccone G, et al. Multi-institutional randomized phase II trial of gefitinib for previously treated patients with advanced non-small-cell lung cancer (the IDEAL 1 trial). J Clin Oncol 2003;21:2237-46.

22. Peus D, Hamacher L, Pittelkow MR. EGF-receptor tyrosine kinase inhibition induces keratinocyte growth arrest and terminal differentiation. J Invest Dermatol 1997;109:751-6.

23. Woodworth CD, Michael E, Marker D, et al. Inhibition of the epidermal growth factor receptor increases expression of genes that stimulate inflammation, apoptosis, and cell attachment. Mol Cancer Ther 2005;4:650-8.

24. Zhang L, Ma S, Song X, et al. Gefitinib versus placebo as maintenance therapy in patients with locally advanced or metastatic non-small-cell lung cancer (INFORM;C-
TONG 0804): a multicentre, double-blind randomised phase 3 trial. Lancet Oncol 2012;13:466-75.

25. Wu YL, Lee JS, Thongprasert S, et al. Intercalated combination of chemotherapy and erlotinib for patients with advanced stage non-small-cell lung cancer (FASTACT-2): a randomised, double-blind trial. Lancet Oncol 2013;14:777-86.

26. Wang J, Wu Y, Dong M, et al. Observation of hepatotoxicity during long-term gefitinib administration in patients with non-small-cell lung cancer. Anti-cancer drugs 2016;27:245-50.

27. Takimoto T, Kijima T, Otani Y, et al. Polymorphisms of CYP2D6 gene and gefitinib-induced hepatotoxicity. Clin Lung Cancer 2013;14:502-7.

28. Swaisland HC, Cantarini MV, Fuhr R, et al. Exploring the relationship between expression of cytochrome P450 enzymes and gefitinib pharmacokinetics. Clin Pharmacokinet 2006;45:633-44.

29. Chhun S, Verstuyft C, Rizzo-Padoin N, et al. Gefitinibphenytoin interaction is not correlated with the C-erythromycin breath test in healthy male volunteers. $\mathrm{Br}$ J Clin Pharmacol 2009;68:226-37.

30. Ruan CJ, Liu DY, Jiang J, et al. Effect of the CYP2C19 genotype on the pharmacokinetics of icotinib in healthy male volunteers. Eur J Clin Pharmacol 2012;68:1677-80.

31. Hirsch FR, Sequist LV, Gore I, et al. Long-term safety and survival with gefitinib in select patients with advanced non-small cell lung cancer: Results from the US IRESSA Clinical Access Program (ICAP). Cancer 2018;124:2407-14.

32. Li L, Zhong W, Liao M, et al. A study on the long-term non-small cell lung cancer survivors in the Expand Access Program of gefitinib in China. Zhongguo Fei Ai Za Zhi 2012;15:332-9.

33. Becker A, van Wijk A, et al. Side-effects of long-term administration of erlotinib in patients with non-small cell lung cancer. J Thorac Oncol 2010;5:1477-80.

34. Manteuffel M, Williams S, Chen W, et al. Influence of patient sex and gender on medication use, adherence, and prescribing alignment with guidelines. J Womens Health (Larchmt) 2014;23:112-9.

Cite this article as: Zhang $\mathrm{W}$, Zhang $\mathrm{Y}$, Zhao Q, Liu X, Chen L, Pan H, Li Y, Lu Y, Huang J, Zhang Z, Du K, Zhang S, Li T, Lu L, Yu G, Wang Y, Yuan X, Yang M, Ma Y, Tan F. Long-term safety of icotinib in patients with non-small cell lung cancer: a retrospective, real-world study. J Thorac Dis 2020;12(3):639-650. doi: $10.21037 /$ jtd.2019.12.115 